\title{
Implications for High Intensity Cycle Ergometer Power Assessment: Morphological and Metabolic Factors
}

\section{Julien S Baker ${ }^{1 *}$ and Lon Kilgore ${ }^{2}$}

${ }^{1}$ Institute of Clinical Exercise \& Health Science, Chair Health \& Exercise Subject Development Group, University of the West of Scotland, Hamilton, Lanarkshire, Scotland, UK ${ }^{2}$ Scottish Sport Academy, Insititute of Clinical Exercise \& Health Science, University of the West of Scotland, Hamilton, Lanarkshire, Scotland, UK

For all force velocity relationships in humans, morphological factors contribute to force and power measurements, and may bias or improve power profiles [1]. Morphological factors that relate to differences in size and structure of lever arms include length and pennation angle of muscle fibres. Force velocity relationships are also interrelated to factors that modify longer duration performances such as the efficiency of oxygen utilisation, muscular blood flow and perceived exertion [2].

Power, the composite product of two factors (force and speed) can incorporate an infinite number of values. Therefore, a range of results are possible with varying contributions from both factors, especially when the criterion is optimisation of absolute maximal power [3]. This suggestion has been substantiated by Baker et al. [4]. A greater power output was achieved during a total body mass (TBM) and fat free mass (FFM) optimisation protocol by increasing both the applied forces and increasing the number of pedal revolutions. With the increasing load, recruitment of more motor units with more muscle fibres per motor unit is most important until the load becomes too heavy [5]. Maximal muscular tension can be produced when the muscle is lengthened, and it declines during the concentric phase of muscle contraction.

Within the range of force velocity interrelationships, those associated with maximised short-term power would be expected to most closely approximate the maximum single contraction as defined by the initial force velocity curve of Hill [6]. Deviations from this relationship are mostly due to fatigue, and the necessary muscular coordination associated with repetitive high frequency motion. The intersubject differences observed between the TBM and FFM protocols may be related to individual inability to generate high levels of velocity. There may be many reasons for this including the proportion of fast twitch fibres (type II) in the exercising muscle, and differences in physiological and biochemical factors that relate to both genetics, and training status.

Type II fibres are known to have faster contraction times and rates of tension development than slow twitch (type I) fibres and are more dependent on glycolysis to maintain ATP rather than the slower process of oxidative phosphorylation [7]. Thorstensson et al. [8] have confirmed a greater proportion of type II fibres in athletes engaged in activities requiring short lived or sprint type power development. In the classical early experiments describing the effects of contraction time on the work and efficiency of the elbow flexors [9] and quadriceps group during cycling [10], it was demonstrated that brief maximal and submaximal contractions were more associated with an increased waste of potential energy than sustained contractions.

In a system performing mechanical work, where heat is liberated and free energy wasted, relatively more free energy must be supplied to maintain performance [11]. Baker et al. [4] suggested that during both a TBM and a FFM protocol, the Peak Power Output (PPO) values obtained were recorded with energy supplied almost exclusively from the degradation of phosphocreatine (PCr) and glycolysis.

Willkie, [12] demonstrated that in muscle, the breakdown of $\mathrm{PCr}$ and glycogen over a cycle of relaxation and contraction is directly proportional to the sum of the heat and work produced. Moreover, during contraction, heat production is at a maximum under conditions in which the work is maximal [13]. Baker et al. [4] indicated that during the initial stages of performance, the work production was greatest when the subjects were optimised for FFM. This suggests a greater or more efficient utilisation of muscle phosphagens when FFM is compared to TBM. In most cases, the time to PPO increased when the subjects were optimised for FFM, indicating a possible alteration in energy system contribution, with glycolysis being used to a lesser extent in the early stages of this resistive force protocol.

This may also indicate an increased degradation of PCr and glycogen and greater changes in metabolic substrates. These factors could have exerted inhibiting effects on the biochemical processes associated with muscle contraction, and may contribute to the fatigue profiles observed. Increased $\mathrm{H}^{+}$in muscle may decrease force generation by impairing $\mathrm{Ca}^{2+}$ release from the sarcoplasmic reticulum [14], or by disturbing cross bridge formation.

High levels of blood acidity and lactate accumulation are also observed following maximal exercise [15]. At high rates of contraction, there is less time for the dispersion of metabolites from muscle, and the intramuscular accumulation of waste products may proceed at an accelerated rate [16]. Results using animal studies have demonstrated that individual fast twitch motor units, and whole muscles with a high percentage of type II fibres, are capable of higher levels of tetanic tension, and are more susceptible to fatigue, than type I fibres [17]. Studies on intact human muscles have reported that individuals with muscles containing a high proportion of type II fibres are capable of faster contraction velocities, and therefore greater force output [8], but are more prone to fatigue during repeated dynamic contraction.

Nilsson et al. [18] demonstrated a strong correlation $(P<0.05)$ between an increase in the ratio of electromyography activity to power associated with fatigue, with a high percentage of type II fibres, suggesting that diminished force was due to a selective drop out of this type of fibre. di Prampero [19] has suggested that a reduction in contractile speed rather than the depletion of high-energy phosphates may be a major cause of fatigue during activities requiring maximal power output. Maximising power output during short duration cycle ergometry is further complicated by the circular motion of the pedals. The circular motion affects the nature of force application, which is influenced by the degree of skill and co-ordination required for a given motion sequence frequency [20].

*Corresponding author: Julien S Baker, PhD, FRSM, FHBA, FIBiol, CBiol CSci, MICR, Director, Institute of Clinical Exercise \&Health Science, Chair Health \& Exercise Subject Development Group, University of the West of Scotland, Hamilton, Lanarkshire, Scotland, UK, Tel: 01698 283100; Fax: 01698 894404; E-mail: jsbaker@uws.ac.uk

Received June 27, 2012; Accepted June 29, 2012; Published June 30, 2012

Citation: Baker JS, Kilgore L (2012) Implications for High Intensity Cycle Ergometer Power Assessment: Morphological and Metabolic Factors. J Sports Med Doping Stud 2:e117. doi:10.4172/2161-0673.1000e117

Copyright: (c) 2012 Baker JS, et al. This is an open-access article distributed under the terms of the Creative Commons Attribution License, which permits unrestricted use, distribution, and reproduction in any medium, provided the original author and source are credited. 
Citation: Baker JS, Kilgore L (2012) Implications for High Intensity Cycle Ergometer Power Assessment: Morphological and Metabolic Factors. J Sports Med Doping Stud 2:e117. doi:10.4172/2161-0673.1000e117

Page 2 of 2

It has also been demonstrated that the internal work associated with the acceleration and deceleration of the leg mass increases with the square of the increased pedalling rate [21]. Therefore, the energy loss at $80 \mathrm{rpm}$ already amounts to $5 \%$ of the external power output and would exceed $20 \%$ at $120 \mathrm{rpm}$. The increase in power output observed when the subjects were optimised for FFM may be the result of increased voluntary command of the supra spinal centres.

This greater contribution may increase fibre recruitment, by the optimisation of individual motor unit firing frequency, and by the synchronisation of the firing patterns between the motor units themselves [22]. This increase depends on the muscles' ability to translate high frequency impulse excitation through the various excitation processes with minimal time delay. In addition, the muscle needs to associate and dissociate the actin and myosin as they repeatedly rotate through successive cross bridge cycles. It is possible, that an increase in neural stimulation will enhance recruitment frequency of the muscle spindles, which would result in a corresponding increase in muscular contraction. The results recorded for the FFM protocol indicate that existing optimisation protocols should be reviewed if increased power output is desirable. Increased peak power output values resulting from higher pedalling rates during optimisation procedures for FFM, may maximise muscle contraction dynamics. These findings are in contrast with previous authors $[23,24]$. However, other researchers [4,2529] have found that during high intensity cycle ergometry the power profiles generated are related to the subjects FFM or to the mass of the muscles that perform the test.

This suggests that the maximal power outputs obtainable, and the relative contribution of the energy systems involved during experimental high intensity cycle ergometer exercise, need further evaluation. Procedures producing realistic power values, that relate to the active muscle tissue utilised during this type of exercise, may need to be explored in preference to methods that are inclusive of both lean and fat mass.

\section{References}

1. Bosco C, Komi PV (1979) Mechanical characteristics and fibre composition of human leg extensor muscles. Eur J Appl Physiol Occup Physiol 41: 275-284.

2. Pugh LG (1974) The relation of oxygen intake and speed in competition cycling and comparative observations on the bicycle ergometer. J Physiol 241: 795808.

3. Inbar O, Bar-Or O, and Skinner S (1996) The Wingate Anaerobic Test. Leeds: Human Kinetics.

4. Baker JS, Bailey DM, Davies B (2001) The relationship between total-body mass, fat-free mass and cycle ergometry power components during 20 seconds of maximal exercise. J Sci Med Sport 4: 1-9.

5. Åstrand PO, Rodahl K (1986) Textbook of Work Physiology. McGraw Hill Book Company.

6. Hill AV (1938) The heat of shortening and the dynamic constant of muscle. Proceedings of the Royal Society: 126: 136-195.

7. McCartney N, Heigenhauser GJ, Jones NL (1983) Power output and fatique of human muscle in maximal cycling exercise. J Appl Physiol 55: 218-224.

8. Thorstensson A, Sjödin B, Karlsson J (1975) Enzyme activities and muscle strength after "sprint training" in man. Acta Physiol Scand 94: 313-318.

9. Hill AV (1922) The maximum work and mechanical efficiency of human muscles, and their most economical speed. J Physiol 56: 19-41.

10. Dickinson S (1929) The efficiency of bicycle-pedalling, as affected by speed and load. J Physiol 67: 242-255.

11. Wilkie DR (1960) Man as a source of mechanical power. Ergonomics 3: 1-8.

12. Wilkie DR (1968) Heat work and phosphorylcreatine break-down in muscle. J Physiol 195: 157-183.
13. Fenn WO, Marsh BS (1935) Muscular force at different speeds of shortening J Physiol 85: 277-297.

14. Nakamura Y, Schwartz A (1972) The influence of hydrogen ion concentration on calcium binding and release by skeletal muscle sarcoplasmic reticulum. $J$ Gen Physiol 59: 22-32

15. Harris RC, Sahlin K, Hultman E (1977) Phosphagen and lactate contents of $m$ quadriceps femoris of man after exercise. J Appl Physiol 43: 852-857.

16. Grimby G, Saltin B (1977) Muscle training. Concluding remarks and actual problems. Lakartidningen 74: 3899-3900.

17. Vandewalle H, Pérès G, Monod H (1987) Standard anaerobic exercise tests. Sports Med 4: 268-289.

18. Nilsson J, Tesch P, Thorstensson A (1977) Fatigue and EMG of repeated fast voluntary contractions in man. Acta Physiol Scand 101: 194-198.

19. di Prampero PE (1981) Energetics of muscular exercise. Rev Physiol Biochem Pharmacol 89: 143-222.

20. Soden PD, Adeyefa BA (1979) Forces applied to a bicycle during norma cycling. J Biomech 12: 527-541.

21. Kaneko M, Yamazaki T (1978) Internal mechanical work done due to velocity changes of the limb in working on a bicycle ergometer, (eds) International series on biomechanics, Vol Ila. University Park press, Baltimore. 86-92.

22. MacDougall JD, Wenger HA, Green HJ (1991) Physiological Testing of the High Performance Athlete. Champaign. IL: Human Kinetics.

23. Katch V (1974) Body weight, leg volume, leg weight and leg density as determiners of short duration work performance on the bicycle ergometer. Med Sci Sports 6: 267-270

24. Patton JF, Murphy MM, Frederick FA (1985) Maximal power outputs during the Wingate anaerobic test. Int J Sports Med 6: 82-85.

25. Van Mil E, Schoeber N, Calvert RE, Bar-or O (1996) Optimisation of force in the Wingate Test for children with a neuromuscular disease. Med Sci Sports Exerc 28: 1087-1092.

26. Blimkie CJ, Roache P, Hay JT, Bar-Or O (1988) Anaerobic power of arms in teenage boys and girls: relationship to lean tissue. Eur J Appl Physiol Occup Physiol 57: 677-683.

27. Doré E, Bedu M, França NM, Van Praagh E (2001) Anaerobic cycling performance characteristics in prepubescent, adolescent and young adult females. Eur J Appl Physiol 84: 476-481.

28. Baker JS, Bailey DM, Dutton J, Davies B (2003) Catecholamine responses to high intensity cycle ergometer exercise: body mass or body composition? J Physiol Biochem 59: 77-83.

29. Baker JS, Bailey DM, Hullin D, Young I, Davies B (2004) Metabolic implications of resistive force selection for oxidative stress and markers of muscle damage during $30 \mathrm{~s}$ of high-intensity exercise. Eur J Appl Physiol 92: 321-327. 